\title{
Surface wave predictions in weakly nonlinear directional seas
}

\author{
Abushet Simanesew ${ }^{\mathrm{a}}$, Karsten Trulsen ${ }^{\mathrm{a}, *}$, Harald E. Krogstad ${ }^{\mathrm{b}}$, José Carlos \\ Nieto Borge ${ }^{\mathrm{c}}$ \\ ${ }^{a}$ Department of Mathematics, University of Oslo, PO Box 1053 Blindern, NO-0316 Oslo, \\ Norway \\ ${ }^{b}$ Department of Mathematical Sciences, NTNU, NO-7491 Trondheim, Norway \\ ${ }^{c}$ Signal Theory and Communications Department, Superior Polytechnic School, University \\ of Alcalá, Alcalá de Henares, 28805, Madrid, Spain
}

\begin{abstract}
We have employed laboratory and numerical experiments in order to investigate propagation of waves in both long and short-crested wave fields in deep water. For long-crested waves with steepness, $\epsilon=k_{c} a_{c}=0.1$ (a fairly extreme case), reliable prediction can be performed with the modified nonlinear Schrödinger equation up to about 40 characteristic wavelengths. For short-crested waves the accuracy of prediction is strongly reduced with increasing directional spread.
\end{abstract}

Keywords:

\section{Introduction}

Predictions are needed in many areas of science and technology. Applications range from the weather forecast to the financial market and the height of the incoming waves that hit you in the sea. If we should propose a rough 5 categorization one could say that the prediction is stochastic if repeated cases with the same input give different output due to some inherent randomness in such models. If identical input produces the same output one may talk about deterministic prediction. Deterministic prediction reaches the final state from the initial state by applying a deterministic propagation operator.

\footnotetext{
* Corresponding author

Email address: karstent@math.uio.no (Karsten Trulsen)
}

Preprint submitted to Journal of Applied Ocean Research

March 20, 2017 
The physics of ocean waves has been thoroughly studied for many years, and provides an excellent basis both for stochastic and deterministic prediction on various spatial and temporal scales. In particular, the surface is weakly nonlinear with the leading order contribution being a space/time Gaussian field. Analysis of surface wave problems has led to a large number of nonlinear wave 15 equations, some of which are used as propagators in the deterministic predictions studied below.

Dynamic positioning of vessels during sensitive offshore operations such as float-over installation, equipment lifting, LNG loading connection, ROV operations and helicopter take-off and landing will benefit from a real-time prediction of the local wave conditions in an order of seconds to a few minutes ahead. Real time prediction could also warn against freak waves and could enable a helmsman to decide how to maneuver vessels during dangerous situations within a limited time horizon (e.g. Clauss et al., 2007, 2008, 2009). Another important application is enhanced extraction of ocean wave energy, where the real-time dynamic control of floating wave energy converters can benefit from the possibility of real-time prediction of excitation force into the future (Fusco and Ringwood, 2012).

The majority of existing commercial systems rely on linear wave theory for prediction of the encountering waves. Obtaining input data in the open ocean 30 with the quality demanded by nonlinear wave propagation models is currently not an easy task. Nevertheless, it is possible to obtain high quality input data in a wave basin, for instance, with arrays of several wave probes (Naaijen et al., 2009). Much work has been done to validate nonlinear wave propagation models with long-crested wave experiments in a laboratory. Shemer et al. (1998) stud-

35 ied evolution of unidirectional nonlinear wave groups in a wave tank with the cubic Schrödinger (NLS) equation (Zakharov, 1968; Hasimoto and Ono, 1972). They observed in deep as well as in relatively shallow water experiments that the NLS equation is able to capture the overall features of nonlinear wave group evolution. Trulsen and Stansberg (2001) applied both the NLS and the modified nonlinear Schrödinger (MNLS) equation (Dysthe, 1979; Trulsen and Dysthe, 
1997) in order to investigate the spatial evolution of unidirectional bichromatic waves in a wave tank for deep water. Certain wave properties, which could not be adequately described by the NLS, were successfully captured by the MNLS. They suggested that the MNLS can be used to predict the evolution of long-crested waves at least up to the dimensionless fetch $\epsilon^{2} k_{c} x=3$ compared to $\epsilon^{2} k_{c} x=1$ for NLS and linear wave theory, where $k_{c}$ is the characteristic wavenumber and $x$ is the evolution distance. Trulsen (2003, 2005) later extended the consideration to evolution of irregular waves. The overall conclusions were similar to Trulsen and Stansberg (2001), the main difference being reduced prediction horizon for the irregular wave experiments. Moreover, Trulsen (2003) suggested a theoretical prediction range of the MNLS equation for various sea states.

Unlike many other nonlinear wave propagation models, Schrödinger equations are computationally efficient, but at the cost of a constraint in the spectral bandwidth. This prompted Shemer et al. (2001, 2002) to investigate evolution of an initially broad spectrum that formally violates the assumptions behind the derivation of (M)NLS. They compared experimental results with simulations based on the unidirectional Zakharov equation (Zakharov, 1968) accurate to the third-order (fourth-order) in wave steepness and the NLS (MNLS) equations. For a sufficiently narrow spectrum, the Schrödinger equation yields good agreement with the Zakharov equation. However, for relatively broader initial spectrum the accuracy degenerates compared with the Zakharov equation, which is free of bandwidth constraints. A recent comparison between experiments of broad-banded unidirectional waves and the Zakharov equation show satisfactory agreement (Shemer et al., 2007) suggesting the Zakharov equation as an effective computational tool for prediction. Shemer and Dorfman (2008) reported comparison of experiments of unidirectional wave groups with both spatial and temporal version of the MNLS equation. In addition to good agreement between experiments and simulations, they observed a gradual transformation of an initially symmetric spectrum into a strongly asymmetric one, this is in agreement with Dysthe et al. (2003). 
Until recently, relatively few validation efforts have been made on nonlinear directional wave fields. Some of these describe direct large-scale phase-resolved computations of nonlinear ocean wave fields (Wu et al., 2007; Xiao et al., 2009). However, simulation of typical nonlinear ocean wave fields with this method requires long computation time, limiting its applicability for short-time wave prediction. Direct comparisons of numerical simulations based on Higher-Order Spectral method to wave tank experiments can be found in Ducrozet et al. (2012).

In this paper, we shall consider the prediction of both long and short-crested wave experiments with linear and nonlinear equations. We use bichromatic and irregular waves from Marintek to validate long-crested wave models (Trulsen, 2005). We also use experiments from MARIN to validate directional seas (Naaijen et al., 2009). Among our selection of models, and based on an ensem-

85 ble average of several realizations, the MNLS equation gives better prediction of the experiments for long fetch. For the irregular long-crested wave experiments with steepness $\epsilon=k_{c} a_{c}=0.1$ we achieve reliable prediction over a fetch of about 40 characteristic wavelengths. For short-crested wave experiments we find less accurate prediction for the same fetch.

\section{Prediction theory}

\subsection{Linear least squares prediction (LSQ)}

We first consider a rather simple case of predicting the surface elevation. It is based on a model which is calibrated using data both from observation and prediction locations, which may then be validated on a new set of data. Assume $\mathrm{X}$ denotes the input observation with dimension $O \times N$, where $O$ is number of observation locations and $N$ is the number of discrete times. The output $Y$ denotes the prediction, in the simplest case, for only one prediction location it has the dimension $1 \times N$. The expression for the best linear prediction, $Y_{p}$, takes the form

$$
Y_{p}=\mathbf{q} \mathbf{X},
$$


where the weight $\mathbf{q}$ is chosen so as to make the prediction error, $e=Y-\mathbf{q X}$, as small as possible. Here $\mathbf{q}$ is a vector of size $1 \times O$.

The calibration step consists of determining the parameters q, which may then be applied to Eq. 1 so as to obtain a reasonable prediction of $Y, Y_{p}$, for a new sets of observations. The numerical problem thus consists of solving Eq. 1 for q. However, the resulting system of equations may be overdetermined, and the system may not always have an exact solution. The aim is thus to consider the error expression $e=Y-\mathbf{q X}$ and write the least squares problem

$$
\|e\|^{2}=(Y-\mathbf{q X})(Y-\mathbf{q} \mathbf{X})^{\prime} .
$$

If $\mathbf{X}$ has full rank, one may consider solving the so-called normal equations that appear from minimizing the linear least squares problem in Eq. 2

$$
\mathbf{q}=Y \mathbf{X}^{\prime}\left(\mathbf{X X}^{\prime}\right)^{-1}
$$

Even if $\mathbf{X}$ has full rank, the normal equations may be numerically ill-conditioned when the condition number of $\mathbf{X}$ becomes large. Numerical treatment, such as QR-factorization of $\mathbf{X}$ is thus necessary to avoid numerical problems, (see, e.g. Nocedal and Wright, 2000; Golub and Van Loan, 2013).

Consider $X(t)$ and $Y(t)$ are measurements from two locations, where $Y$ is delayed in time relative to $X$ by $t_{0}$. In this case, $Y$ should be compensated for the forward shift in time compared to $X$, say $Y=\eta\left(t+t_{0}\right)$, where $\eta$ is the surface displacement at the selected location. The optimal time-shift, $t_{0}$ is determined such that the prediction error is minimized.

\subsection{Predictions based on covariance matrices (COV)}

This method is a generalization of the LSQ method. In addition, we shall now allow several simultaneous predictions. Assume that $\mathbf{X}$ and $\mathbf{Y}$ are real or complex multivariate stochastic variables with dimensions $O \times N$ and $M \times N$ respectively, where $M$ is the number of prediction locations. In order to keep things simple, we shall in the following assume that all variables have zero mean. For this method, the prediction $\mathbf{Y}_{p}$ also takes the form given in Eq. 1 where in 
this case $\mathbf{Y}_{\mathbf{p}}$ is a vector, and $\mathbf{q}$ is an $M \times O$-matrix determined by minimizing the error covariance matrix with respect to the partial ordering of positive definite Hermitian matrices (see, Brillinger, 2001, for this elegant technique).

Let $\mathbf{X}$ and $\mathbf{Y}$ have covariance matrices

$$
\Sigma_{\mathbf{X Y}}=\mathrm{E}\left[\mathbf{X Y} \mathbf{Y}^{H}\right]=\Sigma_{\mathbf{Y} \mathbf{X}}^{H}
$$

and similarly for $\Sigma_{\mathbf{X X}}$ and $\Sigma_{\mathbf{Y Y}}$. Superscript $H$ means the Hermitian transposed. Recalling $\mathbf{e}=\mathbf{Y}-\mathbf{Y}_{p}$, the unique minimum for $\Sigma_{\mathbf{e e}}$ is obtained with

$$
\mathbf{q}=\Sigma_{\mathbf{Y X}} \Sigma_{\mathbf{X X}}^{-1}=\Sigma_{\mathbf{X Y}}^{H} \Sigma_{\mathbf{X X}}^{-1}
$$

when $\Sigma_{\mathbf{X X}}$ is nonsingular (Brillinger, 2001, Thm. 8.2.1). The optimal prediction may thus be written as

$$
\mathbf{Y}_{p}=\Sigma_{\mathbf{Y X}} \Sigma_{\mathbf{X X}}^{-1} \mathbf{X}
$$

and the minimal error covariance matrix becomes

$$
\Sigma_{\mathbf{e e}}=\Sigma_{\mathbf{Y Y}}-\Sigma_{\mathbf{X Y}}^{H} \Sigma_{\mathbf{X X}}^{-1} \Sigma_{\mathbf{X Y}}
$$

where the last term on the RHS is the prediction covariance matrix, $\Sigma_{\mathbf{Y}_{p} \mathbf{Y}_{p}}=$ $\Sigma_{\mathbf{X Y}}^{H} \Sigma_{\mathbf{X X}}^{-1} \Sigma_{\mathbf{X Y}} \cdot$ Thus,

$$
\Sigma_{\mathbf{Y Y}}=\Sigma_{\mathbf{Y}_{p} \mathbf{Y}_{p}}+\Sigma_{\mathbf{e e}}
$$

(Brillinger, 2001). In conclusion, we may therefore always decompose $\mathbf{Y}$ into the best linear predictor from $\mathbf{X}$, as shown in Eq. 6, and an orthogonal component, $\mathbf{e}=\mathbf{Y}-\mathbf{Y}_{p}$, which is completely uncorrelated. It is interesting to observe that $\mathbf{e}$ is additive and orthogonal to the prediction of $\mathbf{Y}$. Obviously, should $\mathbf{X}$ be orthogonal to $\mathbf{Y}$, the prediction is simply $\mathbf{Y}_{P}=0$ and $\mathbf{e}=\mathbf{Y}$.

In the case that $\mathbf{X}$ and $\mathbf{Y}$ are Gaussian, a best prediction, $\mathbf{Y}_{\underline{p}}$, always exists and is denoted by the conditional expectation, $\mathbf{E}(\mathbf{Y} \mid \mathbf{X})$ (Øksendal, 2003). Although hard to find in general, when dealing with finite dimensional Gaussian variables the best prediction is simply the familiar multivariate linear regression and the solution is similar to the one above. The prediction error in this case is also uncorrelated to $\mathbf{Y}_{P}$. 


\subsection{Linear wave simulation based on synthetic directional spectrum}

In the following, we present methods handy for the numerical assessment of the effects of directional parameters and the amount of input data on wave predictability. The method combines LWT and the one discussed in Section 2.2 For a more comprehensive treatment of LWT we refer to Kahma et al. (2005) and Goda (2010).

For a weakly stationary and homogeneous stochastic field, the covariance function, $\rho$, of the surface elevation, $\eta$, may be written in terms of the threedimensional $(\mathbf{k}, \omega)$-spectrum,

$$
\rho(\mathbf{r}, t)=\int_{\mathbf{k}, \omega} e^{i(\mathbf{k} \cdot \mathbf{r}-\omega t)} d \chi(\mathbf{k}, \omega) .
$$

where $\chi$ is the three-dimensional spectrum of the surface elevation, $\mathbf{r}=(x, y)$ is the horizontal position vector, $t$ is time, $\mathbf{k}$ is the wavenumber vector and $\omega$ the frequency. The above integral may also be expressed in terms of the wavenumber vector spectrum $\Psi(\mathbf{k})$ by means of the formula,

$$
\rho(\mathbf{r}, t)=\int_{\mathbf{k}} \cos (\mathbf{k} \cdot \mathbf{r}-\omega t) \Psi(\mathbf{k}) \mathrm{d} k_{x} \mathrm{~d} k_{y},
$$

(Kahma et al., 2005). The wavenumber spectrum, $\Psi(\mathbf{k})$, is an integration of $\chi(\mathbf{k}, \omega)$ over frequencies, keeping $\mathbf{k}$ fixed. Further simplification may be achieved by limiting the model to the leading order in wave steepness, which leads to the dispersion relation connecting $\mathbf{k}$ and $\omega$, e.g., $\omega^{2}=g k$ in deep water, where $k=|\mathbf{k}|$. In this case, the integral in Eq. 10 may be expressed in terms of the directional spectrum, $E(\omega, \theta)=S(\omega) D(\theta, \omega)$ :

$$
\rho(\mathbf{r}, t)=\int_{\omega=0}^{\infty} S(\omega) \int_{\theta=0}^{2 \pi} D(\theta, \omega) \cos [k(\omega) r \cos (\theta-\varphi)-\omega t] \mathrm{d} \theta \mathrm{d} \omega,
$$

130

$$
\text { sided frequency spectrum, and } D(\theta, \omega) \text { the directional distribution normalized }
$$
such that $\int_{0}^{2 \pi} D(\theta, \omega) \mathrm{d} \theta=1$.

In order to apply the prediction formulas in Eqs. 5 7 7 , it is necessary to have reliable knowledge of the covariance matrices. In a theoretical LWT setting, the 135 matrices may be computed from Eq. 11, whereas in practice, the matrices need 
to be estimated from the data. The choice is then whether to estimate the directional spectrum and apply that in Eq. 11, or if it is possible, to estimate the covariance matrices directly from the data by ensemble averaging. Estimates of covariance matrices may be obtained from data in a lot of ways, utilizing e.g. their special structure like the Toeplitz matrices for prediction in stationary time series (Burg et al., 1982).

For the numerical investigations reported in Section 4.1, we apply LWT and the directional spectrum for simulation of the covariance function (See Eq. 11). The method is a useful tool for system assessment, but requires stationary and periodic sea state conditions (in time $t$ ) and will experience problems if topography is important. However, for the experimental studies in Section 4.2. Eqs. 48 can be applied regardless of any additional assumptions beyond stationarity. Note that we also apply a similar time-shift $t_{0}$ as described in Section 2.1.

\subsection{Linear deterministic wave prediction (LDWP)}

We shall represent the wavy water surface, $z=\eta(\mathbf{r}, t)$ by linear wave theory, where $z$ is a vertical coordinate. In a Gaussian field, the wave propagation model can be described as a superposition of many monochromatic waves with different frequencies, phases and amplitudes traveling in different directions. We impose periodic boundary conditions for the $y$ - and $t$-coordinates, with periodic intervals of length $L_{y}$ and $T$ respectively. For propagation in the $x$-direction we may write

$$
\eta(\mathbf{r}, t)=\operatorname{Re}\left\{\sum_{j} \sum_{l} c\left(k_{y j}, \omega_{l}\right) e^{i\left(k_{x} x+k_{y j} y-\omega_{l} t\right)}\right\}
$$

where $\omega_{l}=2 \pi l / T, k_{y j}=2 \pi j / L_{y}$ and Re means taking the real part. The $2 D$ Fast Fourier Transform (2D-FFT) of $\eta(x=0, y, t)$ yields the complex coefficients, $c\left(k_{y j}, \omega_{l}\right)$. The wavenumber in the propagation direction $x, k_{x}$, is related to $\omega_{l}$ and $k_{y j}$ by the dispersion relation, e.g., $\left\{k_{x}\right\}_{(j, l)}= \pm \sqrt{\omega_{l}^{4} / g^{2}-k_{y j}^{2}}$ in 


\subsection{Prediction with cubic and modified nonlinear Schrödinger equations}

We shall employ a three-dimensional modified nonlinear Schrödinger model that describes the evolution of the surface elevation indirectly using the evolution of its complex envelope, $B$. In the following, all expressions are nondimensionalized by a characteristic angular frequency $\left(\omega_{c}\right)$ and the corresponding characteristic wavenumber $\left(k_{c}\right)$. We let $\omega_{c}$ be the mean frequency with respect to the power spectrum, i.e.,

$$
\omega_{c}=\frac{\sum_{l} \sum_{j} \omega_{l}\left|\hat{\eta}\left(x=0, k_{y j}, \omega_{l}\right)\right|^{2}}{\sum_{l} \sum_{j}\left|\hat{\eta}\left(x=0, k_{y j}, \omega_{l}\right)\right|^{2}}
$$

and determine $k_{c}$ from the linear dispersion relation in deep water, $k_{c}=\omega_{c}^{2} / g$. Here $\hat{\eta}$ is the Fourier transform of the surface elevation at the observation location. Assuming irrotational flow of an incompressible inviscid fluid, we expand the velocity potential $\phi$ and surface elevation $\eta$ of the free surface in harmonic series

$$
\phi=\bar{\phi}+\operatorname{Re}\left\{A e^{i(x-t)}+A_{2} e^{2 i(x-t)}+A_{3} e^{3 i(x-t)}+\ldots\right\},
$$

and

$$
\eta=\bar{\eta}+\operatorname{Re}\left\{B e^{i(x-t)}+B_{2} e^{2 i(x-t)}+B_{3} e^{3 i(x-t)}+\cdots\right\} .
$$

Here $\bar{\eta}, B_{2}$ and $B_{3}$ are the zeroth, second and third harmonic bound waves. They are slowly varying functions of space and time given by

$$
\bar{\eta}=-\frac{\partial \bar{\phi}}{\partial t}, \quad B_{2}=\frac{1}{2} B^{2}+i B \frac{\partial B}{\partial t}, \quad B_{3}=\frac{3}{8} B^{3} .
$$

Provided that the steepness is small $\epsilon=k_{c} a_{c} \ll 1$, the bandwidth is narrow $|\Delta \mathbf{k}| / k_{c}=\mathcal{O}(\epsilon)$, the depth is large $\left(k_{c} h\right)^{-1}=\mathcal{O}(\epsilon)$, and neglecting terms of relative order higher than $\epsilon^{4}$, we get the modified nonlinear Schrödinger (MNLS) equation. Here $a_{c}=\sqrt{2 \mathrm{E}\left[\eta^{2}\right]}$ is the characteristic amplitude, $\Delta \mathbf{k}$ is a characteristic modulation wave vector relative to the characteristic wave vector and $h$ is depth (Trulsen, 2005). The potential $\bar{\phi}$ of the induced mean flow is governed 
by the equations

$$
\begin{aligned}
\frac{\partial \bar{\phi}}{\partial z} & =-\frac{\partial|B|^{2}}{\partial t} \quad \text { at } \quad z=0, \\
4 \frac{\partial^{2} \bar{\phi}}{\partial t^{2}}+\frac{\partial^{2} \bar{\phi}}{\partial y^{2}}+\frac{\partial \bar{\phi}}{\partial z^{2}} & =0 \quad \text { for } \quad-h<z<0, \\
\frac{\partial \bar{\phi}}{\partial z} & =0 \quad \text { at } \quad z=-h .
\end{aligned}
$$

The first, second and third harmonic complex amplitudes of the potential, $A$, $A_{2}$ and $A_{3}$, will not be considered here. The spatial evolution form of the modified nonlinear Schrödinger (MNLS) equation (Dvsthe, 1979; Brinch-Nielsen and Jonsson, 1986; Trulsen and Dysthe, 1997; Toffoli et al., 2010) with exact linear dispersion is given by

$$
\frac{\partial B}{\partial x}+\mathcal{L} B+i|B|^{2} B-\left[8|B|^{2} \frac{\partial B}{\partial t}+2 B^{2} \frac{\partial B^{*}}{\partial t}+4 i \frac{\partial \bar{\phi}}{\partial t} B\right]=0,
$$

(Trulsen et al., 2000; Trulsen, 2005). The superscript $*$ means complex conjugate. The terms in the bracket should be discarded to recover the cubic nonlinear Schrödinger (NLS) equation, while the first two terms comprise the linear Schrödinger (LS) equation. The linear dispersive part, in its exact form is defined by the operator $\mathcal{L}$ as

$$
\mathcal{L}=-i\left\{\left[\left(1+i \frac{\partial}{\partial t}\right)^{4}+\frac{\partial^{2}}{\partial y^{2}}\right]^{1 / 2}-1\right\}
$$

In agreement with the accuracy of the nonlinear truncation, for MNLS all terms in Eq. 15]are used, for NLS only first and second-order terms are retained, and for LS only first-order terms are retained.

Ocean wave fields are generally non-periodic. However, since our numerical techniques depend heavily on representation with Fourier transform, we assume periodic boundary conditions for the $y$-direction and for time. The discrete Fourier representation of the first harmonic complex amplitude is

$$
B\left(x=0, y_{m}, t_{n}\right)=\sum_{l} \sum_{j} \hat{B}\left(x=0, k_{y j}, \omega_{l}\right) e^{i\left(k_{y j} y_{m}-\omega_{l} t_{n}\right)}
$$

160

where $y_{m}$ and $t_{n}$ are collocation points in the $y$-direction and time, and $\hat{B}\left(x, k_{u j}, \omega_{l}\right)$ the 2-D Fourier transform of $B$. We employ the numerical method of Lo and Mei 
(1985, 1987) with periodic boundary conditions in time and transverse direction. Evolution in the $x$-direction is achieved with a splitting scheme in which the linear part of Eq. 18 is integrated exactly in Fourier space and the nonlinear part is integrated by finite differences.

The initialization of the Schrödinger equations entails prior determination of $\omega_{c}$ and $k_{c}$ followed by extraction of the first harmonic complex amplitude, $B$. The envelope, $B(x=0, y, t)$, may be extracted from the data by low-pass filtering the Fourier transform of the term on the left hand side of $(2 \eta(x=$ $\left.\left.{ }_{170} x_{0}, y, t\right) e^{i \omega_{c} t}=B+B^{*} e^{-2 i \omega_{c} t}\right)$ around the origin. Here, $\eta(x=0, y, t)$ is a $2-$ D surface displacement which could be used as initial condition for the wave propagation models.

\subsection{Error between predicted and experimental surface elevation}

To quantify the accuracy of predictions in the experiments, we define a normalized prediction error averaged over time,

$$
\operatorname{error}(\mathbf{r})=\sqrt{\frac{\sum_{n}\left(\eta_{\text {pred }}\left(\mathbf{r}, t_{n}\right)-\eta_{\text {exp }}\left(\mathbf{r}, t_{n}\right)\right)^{2}}{\sum_{n}\left(\eta_{\exp }\left(\mathbf{r}, t_{n}\right)\right)^{2}}},
$$

where $\eta_{p r e d}$ and $\eta_{\exp }$ are predicted and experimental surface displacements re-

\section{Descriptions of experiments}

In the following, we present descriptions of Marintek towing tank (bichromatic), Marintek ocean basin (irregular) and MARIN basin (irregular) experiments. In all of the experiments and simulations presented here, we adopt the 
(2005, Fig.2) for scatter plots of $T_{p}$ and $H_{s}$ from the northern North Sea, where curves of constant steepness are also plotted using the definition $\epsilon=$ $\sqrt{2} \pi^{2} H_{s} /\left(g T_{p}^{2}\right)$. This definition is slightly different from the one we used above since $T_{p} \gtrsim T_{c}$. The steepness for the present study, ranging from moderate $(\epsilon=0.05)$ to extreme $(\epsilon=0.12)$ case, are listed in table 1 .

To keep resemblance with the reports provided from the respective laboratories, we evaluate the bichromatic waves at model-scale and the irregular waves at full-scale. However, to ease the interpretations most of our results are presented in normalized form, time as $t / T_{c}$, space as $\left(x / \lambda_{c}, y / \lambda_{c}\right)$ and surface elevation as $k_{c} \eta$.

\subsection{Long-crested seas}

(a)

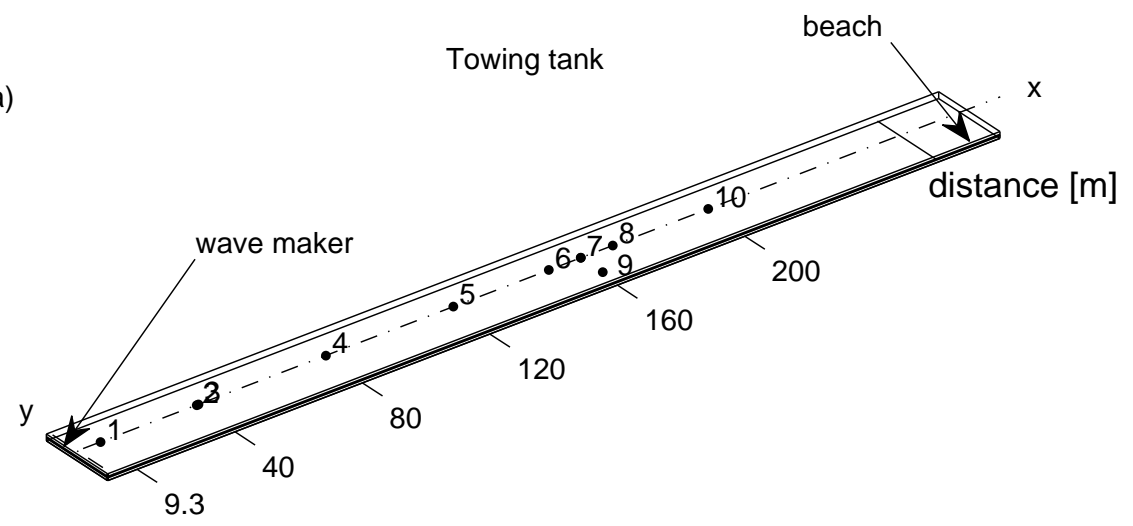

(b)

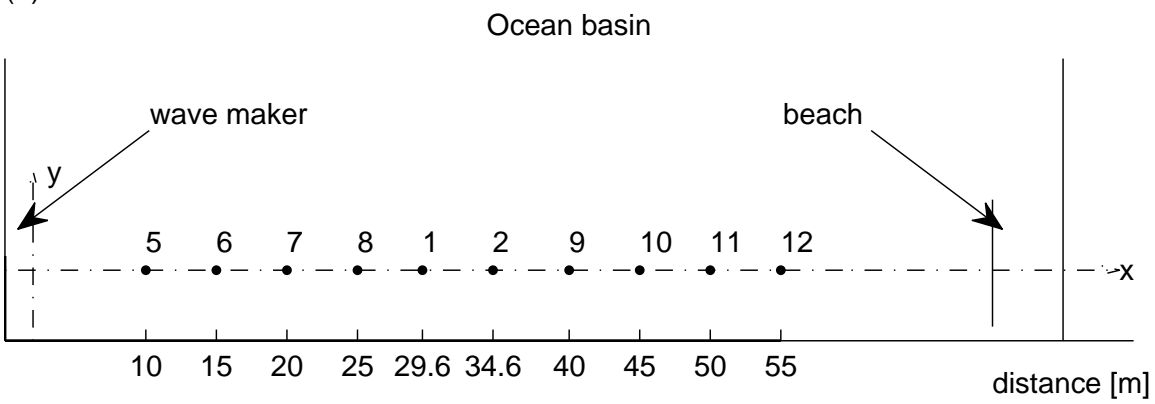

Figure 1: Sketches of Marintek Towing tank (a) and Ocean basin (b) with locations of the wave staffs. 


\subsubsection{Bichromatic waves}

This experiment was performed in the $270 \mathrm{~m}$ long and $10.5 \mathrm{~m}$ wide towing tank at Marintek (Stansberg, 1993, 1995, 1998). The depth of the tank is $10 \mathrm{~m}$ for the first $80 \mathrm{~m}$ and $5 \mathrm{~m}$ otherwise. The surface elevation was measured by 10 wave staffs at different locations simultaneously. Apart from probe 9 all the others were located along the centerline of the tank. A sketch of the wave tank is shown in Fig. 1 (a). The bichromatic wave properties are given in Table1, In this case, the characteristic wavenumber is determined based on the arithmetic mean value of nominal periods of the two wave components, i.e., following the dispersion relation in deep water $k_{c}=\left(2 \pi / T_{c}\right)^{2} / g$, where $T_{c}=\left(T_{1}+T_{2}\right) / 2$. The non-dimensional depth is thus $k_{c} h=11$ for the former and $k_{c} h=5.5$ for the latter section of the wave tank, however, our numerical simulations are based on $k_{c} h=11$ for the entire tank. Since the prediction error due to ignoring the jump at $80 \mathrm{~m}$ in the tank is small, it will not be a primary source of error in the present study. We should also note that the given steepness applies to the portion of the time series at probe 1 used for initialization of the numerical models.

Normalized errors between predicted and measured waves are computed using Eq. 21 at probes 1, 2, 4, 5, 6, 7, 8 and 10 with a non-dimensional time of length 20 , starting from $t / T_{c}=9$ at probe 1 . The normalized errors are then ensemble-averaged over 1200 realizations obtained by moving the window bounded by the two skew lines shown in Fig. 22 The realizations are obtained by partially overlapping time trace sections, separated by a sample size corresponding to $1 / 2 T_{c}$. Since realizations constructed in this way will be dependent, it requires larger ensemble size for sufficient convergence. We refer to Naaijen et al. (2014) for further discussion about the time overlap and the ensemble size required for optimal convergence. In Fig. 2, the slope of the two skew lines corresponds to the magnitude of the LWT group velocity of the characteristic wave. 
Table 1: Summary of Marintek towing tank (bichromatic), Marintek ocean basin (irregular) and MARIN ocean basin (irregular) tests. The Marintek towing tank experiments are given in model scale, whereas all of the ocean basin experiments are given at full scale.

\begin{tabular}{cccccccc}
\hline Wave types & \multicolumn{2}{c}{ Wave periods $[\mathrm{s}]$} & \multicolumn{2}{c}{ Wave heights $[\mathrm{m}]$} & Dir. spread & Steepness \\
& $T_{1}$ or $T_{0}$ & $T_{2}$ & $H_{1}$ or $H_{m 0}$ & $H_{2}$ & $\sigma_{1}\left[^{\circ}\right]$ & $\epsilon$ \\
\hline \hline \multicolumn{2}{c}{ Marintek } \\
Bichromatic & 1.90 & 2.10 & 0.16 & 0.16 & 0 & 0.11 \\
Irregular & 14.0 & - & 14 & - & 0 & 0.12 \\
\hline \multicolumn{2}{c}{ MARIN (irregular) } & & & & & \\
Narrow & 9 & - & 2.5 & - & 6 & 0.05 \\
Medium & 9 & - & 2.5 & - & 12.5 & 0.05 \\
Broad & 9 & - & 2.5 & - & 19 & 0.05 \\
\hline
\end{tabular}

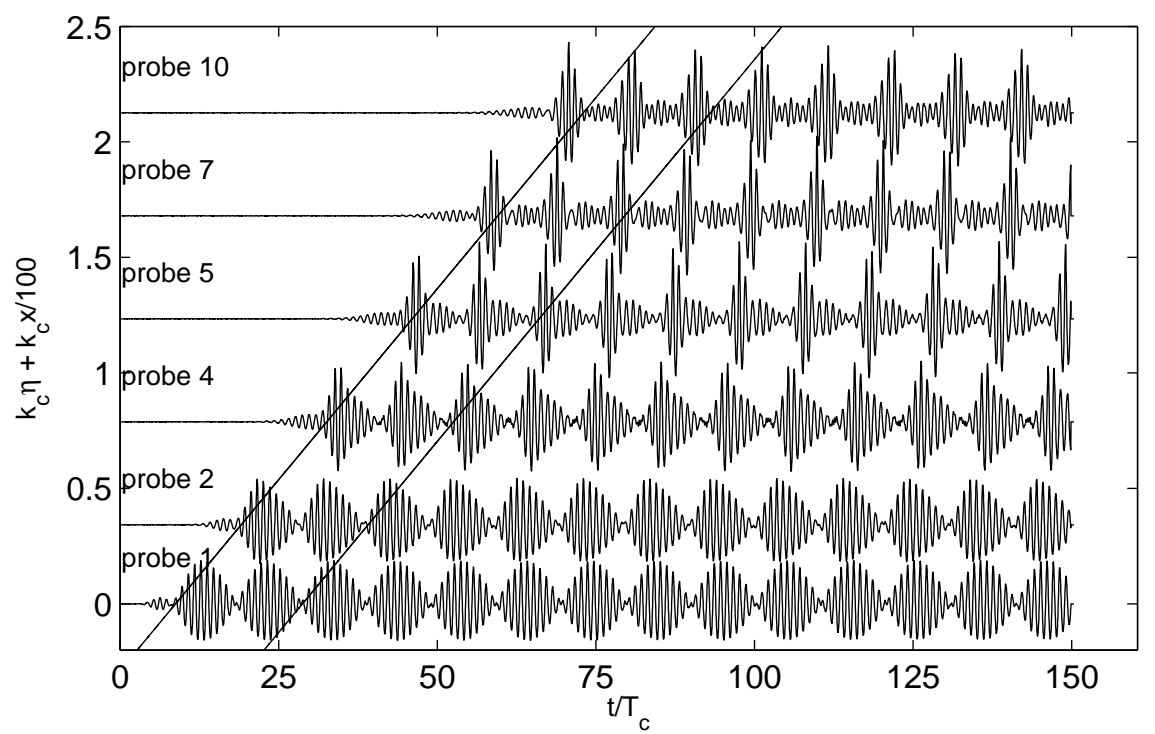

Figure 2: Marintek bichromatic wave records at selected probes along the centerline of the towing tank. The slanted lines, given by the LWT group velocity of the characteristic wave, bound the evolution of wave groups. 


\subsubsection{Irregular waves}

This experiment was performed in the ocean basin at Marintek. The sketch of the wave tank is shown in Fig. 1(b). The wave maker was programmed with an irregular wave spectrum, with peak period $14 \mathrm{~s}$ and significant wave height $14 \mathrm{~m}$ (after scaling with 1:200), see also Table1. We use a non-dimensional time trace of length 1694, starting at $t / T_{c}=25$ of the original time series (See Fig. 3). The measurements at probe 5 are used for initialization of the numerical models. Normalized errors between predicted and measured waves are computed using Eq. 21 at all probes with a non-dimensional time trace of length 14, starting at $t / T_{c}=25$ at probe 5 . The normalized errors are then ensemble-averaged over 800 realizations made by moving the window bounded by the two skew lines shown in Fig. 3. It is worth mentioning that the deviation of the propagation velocity of groups from the linear group velocity $\left(c_{g, l w t}\right)$ seen in Figs. 2 and 3 is a nonlinear effect captured by the MNLS equation(e.g., Trulsen, 1998). The above assumption that the predictable zone is bounded just by $c_{g, l w t}$ is not an optimal choice both for broad-banded and nonlinear waves. There are numerical and experimental evidences that wave groups propagate faster than the linear group velocity (e.g., Trulsen, 1998). Moreover, for broad-banded waves, the group velocity corresponding to the longest and shortest wave components present in the initial time-trace should bound the spatio-temporal prediction horizon (Wu, 2004; Naaijen and Huijsmans, 2010). As a consequence, the two bounding lines will have different slopes, however, we do not incorporate the corresponding effect in the present study.

\subsection{Short-crested seas}

Data for this study were collected by MARIN as part of the OWME project. A detailed description of the facilities and experiments is presented in Naaijen et al. (2009). These experiments were carried out in the $40 \mathrm{~m}$ long, $170 \mathrm{~m}$ wide and $5 \mathrm{~m}$ deep MARIN seakeeping and manoeuvering basin at a scale of $1: 70$. The basin has flap type wave makers on two sides and absorbing beaches on the opposite sides. The wave maker, parallel to the $y$-axis, was programmed with 


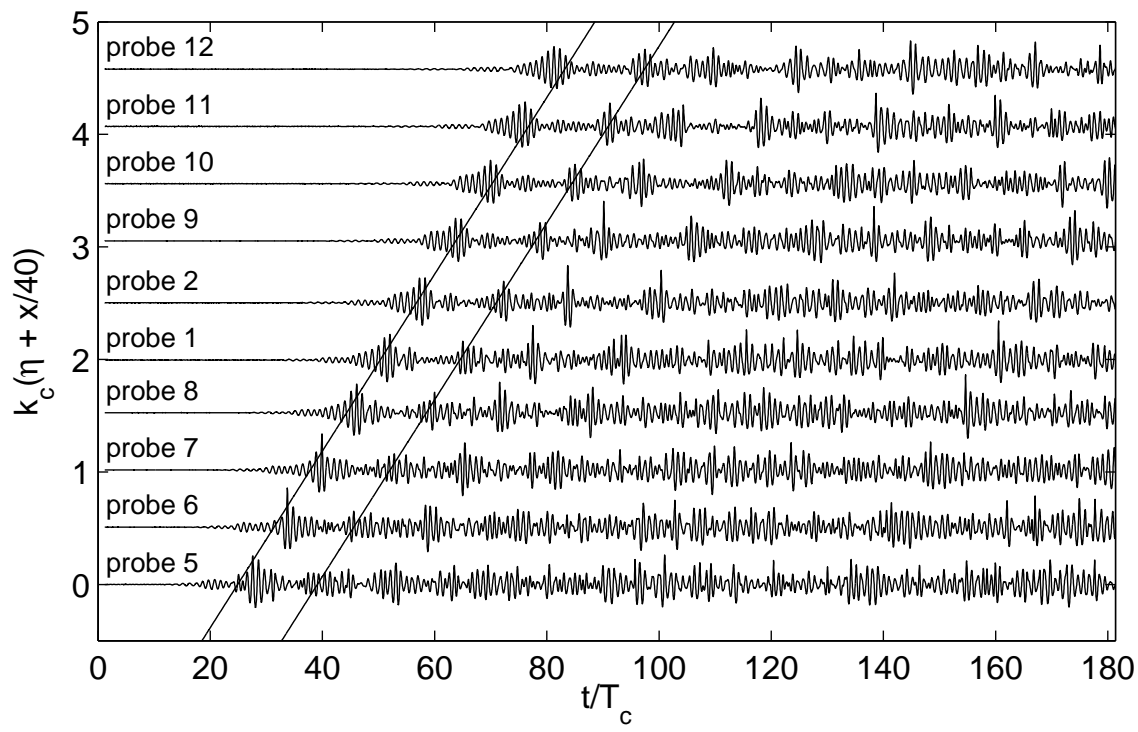

Figure 3: Marintek irregular wave records at all probes in the ocean basin. The slanted lines, given by the LWT group velocity of the characteristic wave, bound the evolution of wave groups.

a directional spectrum which is obtained by multiplying a JONSWAP spectrum and a directional distribution similar to the standard cos- $2 s$ distribution (Longuet-Higgins et al., 1963). The spectra were generated according to the specifications given in table 1, where the three different test cases, ranging from narrow to broad directional spread, are listed. Here, $\sigma_{1}$ is the directional spread (circular standard deviation of the directional distribution (Mardia, 1972)). For further discussion about its functional relation with other wave parameters, we refer to Simanesew et al. (2016) and references therein.

The wave measurements were carried out using a $126 \mathrm{~m}$ by $126 \mathrm{~m}, 10 \times 10$ wave probe array of grid size $14 \mathrm{~m}$ in both directions as shown in Fig. 4a in full-scale. The array was connected to a carriage which could be moved to various locations in the basin between tests. Measurement locations for the present study are shown in Fig. $4 \mathrm{~b}$ The main wave propagation direction was along the $x$-axis. During the test, the input directional distribution was 


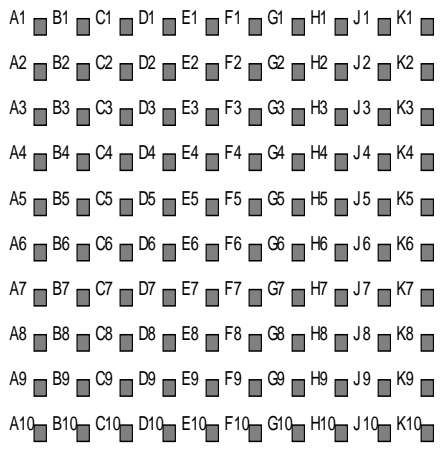

(a) Wave probes arranged in a square-array with sides $126 \mathrm{~m}$ (full scale)

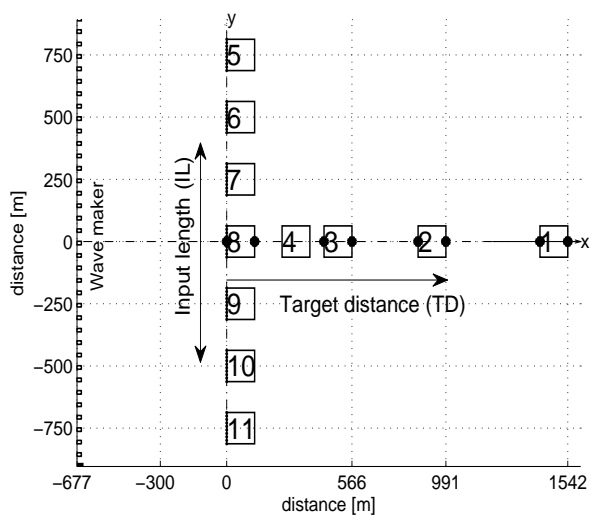

(b) Wave gauge array locations. The nearest measurements are about $677 \mathrm{~m}$ away from the wave maker.

Figure 4: Probe arrangement and wave gauge array locations. In Fig. 4b data along the $y$-axis are used to initialize the models, whereas marked dots are locations where predictions and experiments are compared.

limited to directions within $\left(-15^{\circ}, 15^{\circ}\right)$ for narrow, $\left(-30^{\circ}, 30^{\circ}\right)$ for medium and $\left(-45^{\circ}, 45^{\circ}\right)$ for broad distribution.

We use a non-dimensional time domain of 380 from a total of about 650 starting from $t / T_{c}=50$ (See Fig. 5). Detailed comparisons between predicted and measured waves are made at (locations, probes): (8, A5), (3, K5), (2, K5) and (1, K5) with a non-dimensional time trace of length 28 , starting at $t / T_{c}=144$ at the initial probes, moving with the magnitude of LWT group velocity of the characteristic wave (See Fig. [5). The probes are located at distances $677 \mathrm{~m}, 1243 \mathrm{~m}, 1668 \mathrm{~m}$ and $2219 \mathrm{~m}$ from the wave maker, respectively. Normalized prediction errors are computed using Eq. 21 at selected probes, starting at $t / T_{c}=50$ at location 8 probe $\mathrm{A} 5$ and then ensemble-averaged over 800 realizations.

For the medium wave fields, $\sigma_{1}=12.5^{\circ}$, the models were initialized by the outermost seventy time series located on the left sides of locations 5 to 11 . Grid resolution was set to $14 \mathrm{~m}\left(0.13 \lambda_{c}\right)$ over a length of fifteen characteristic 


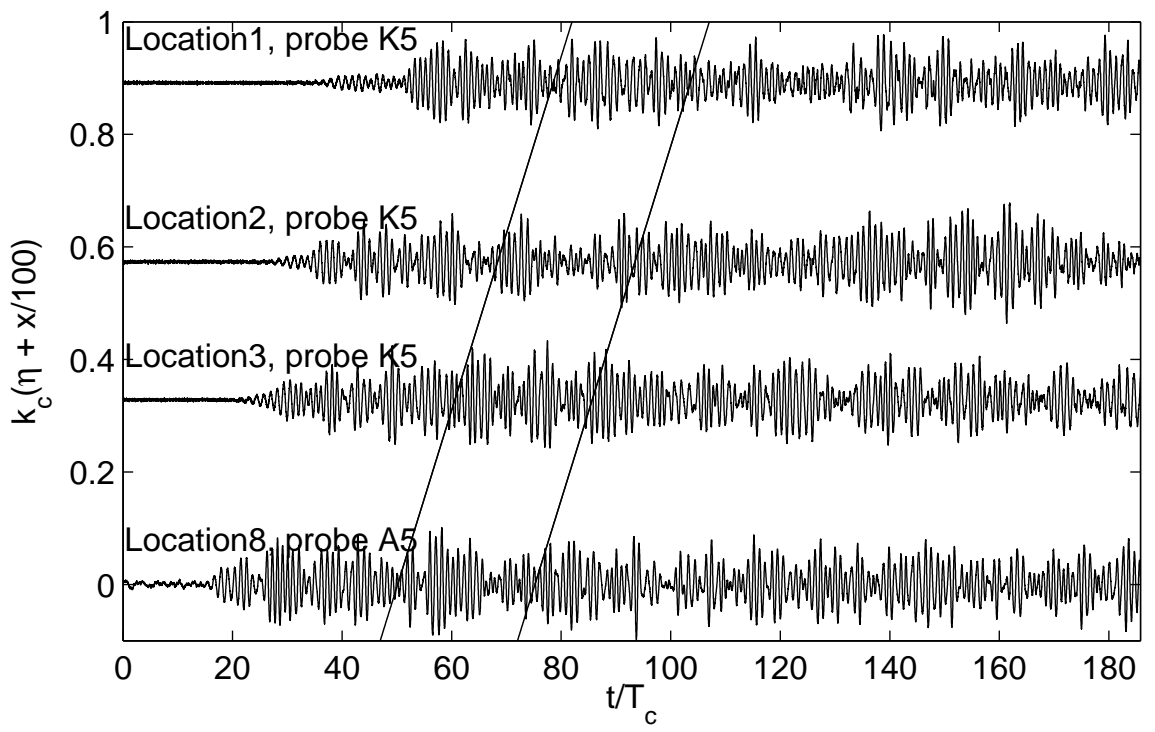

Figure 5: MARIN: Short-crested seas $\left(\sigma_{1}=12.5^{\circ}\right)$ at selected probes. The slanted lines, given by the LWT group velocity of the characteristic wave, bound the evolution of wave groups.

wavelengths $\left(\lambda_{c}\right)$ in the transverse direction. For waves with narrow and broad directional distributions, available measurements were limited to locations 7,8 and 9 covering only six characteristic wavelengths in the transverse direction. This means that the most short-crested sea experiments have insufficient input data to characterize waves that would influence the upstream wave field.

Moreover, due to the finite size of the movable $10 \times 10$-array, the measurements are non-equidistant in the $y$-direction, restricting the use of the standard FFT-algorithm to reconstruct the initial envelope. We thus employed the $2 \mathrm{D}$ bilinear interpolation technique, to provide equidistant measurements for our numerical schemes with a possible trade-off prediction accuracy.

\section{Results}

In this section we present results from numerical and laboratory experiments. We shall consider measured time series at discrete spatial points to initialize our models, and perform predictions forward in time and space. The setting is 
shown in Fig. 6, where the time series, in the initial domain, are distributed uniformly over an interval of length $I L$. It turns out that the prediction error at a target with distance $T D$ depends strongly on parameters such as wave directionality and the ratio between $I L$ and $T D$, alternatively expressed as $\theta_{p}=2 \operatorname{atan}(I L /(2 T D))$.

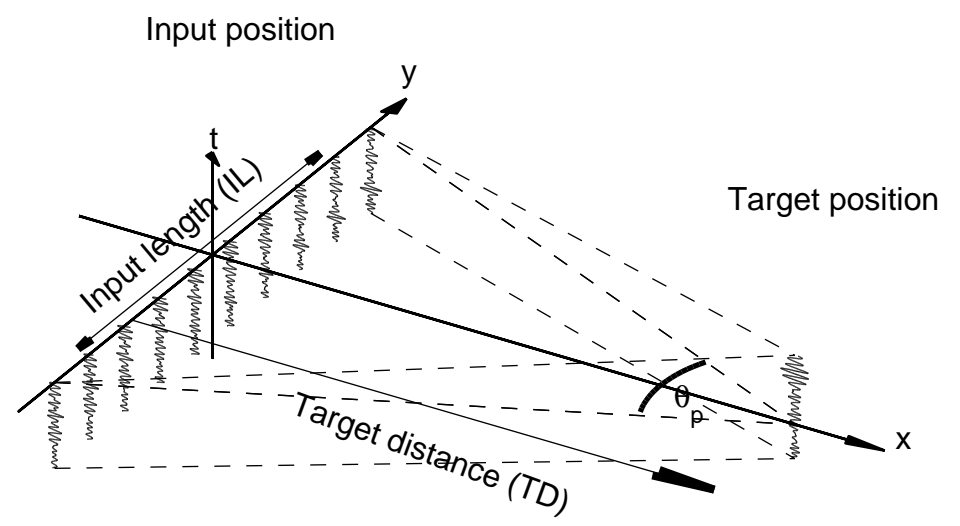

Figure 6: Visualization of directional-wave prediction at a target with distance $T D$ based on input data along a transverse interval of length $I L$. The middle two dashed lines are $t=$ const., whereas the upper and lower curves have slope equal to the magnitude of the LWT group velocity of the characteristic wave.

\subsection{Linear numerical experiments}

In order to investigate the effects of the above parameters, we carried out numerical experiments within the frame of LWT discussed in Section 2.3 We use a JONSWAP spectrum with a peak period, $T_{p}=9 \mathrm{~s}$ and a peakedness factor $\gamma$ of 3.3. Wave directionality is included by the standard cos- $2 s$ distribution with various selection of directional spread. Figure 7 shows contour plots of normalized prediction error variances as defined in Eq. 7 from a single observation in space, located at $(x, y)=(0,0)$. All physical quantities are scaled with the values corresponding the spectral peak. 

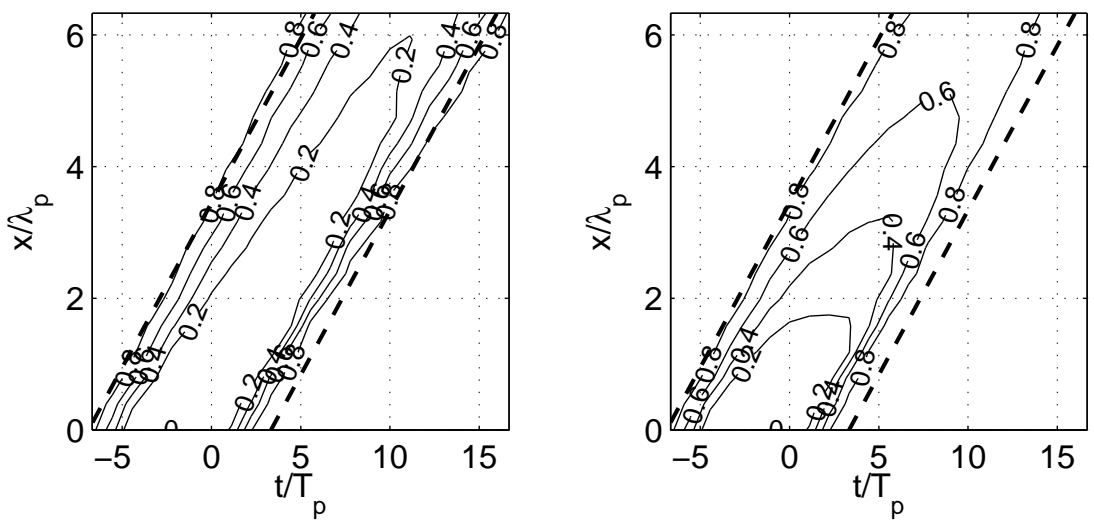

Figure 7: Prediction error variances from simulation with single observation located at $\mathbf{r}=$ $(0,0)$. The directional spread is $6^{\circ}$ for the plot on the left and $19^{\circ}$ for the plot on the right side. The slope of the two bounding dashed lines is given by the magnitude of the LWT group velocity of the peak wave.

For a narrow distribution, with $\sigma_{1}=6^{\circ}$, the spatio-temporal prediction horizon is large, and the prediction error increases slowly both with respect to space and time. For a moderately broad distribution, with $\sigma_{1}=19^{\circ}$, we observe a significant reduction in the overall prediction horizon with a fairly rapid change in the prediction error variances. Figure 8 shows various spectra at about three peak wavelengths away from the observation. The error spectrum clearly takes over the prediction spectrum for large evolution distance, and it is obvious that the effect is enhanced for a relatively broad distribution. The observed large error spectrum in the high-frequency region is predominantly due to canceling out of integrals like (11) when arguments $k r$ or $\omega t$ increase. It is known (Riemann-Lebesgue Lemma) that the Fourier transform of an $L^{1}$ function vanishes at infinity.

Since with only a single observation short-wave prediction may not reach the intended target location, adding more input data in the transverse direction partly reduces the effect as illustrated in Fig. 9 It shows the prediction error variances according to Eq. 7 employing single and triple observations. The space-time prediction horizon has been significantly improved for the sim- 
ulation with triple observations as expected. In the following, we investigate the required size of $I L$ for a given directional spread. The separation distance between transverse data points has been set to $50 \mathrm{~m}$ (approximately equivalent to $\left.0.4 \lambda_{p}\right)$, and distributed uniformly over the length $I L$.
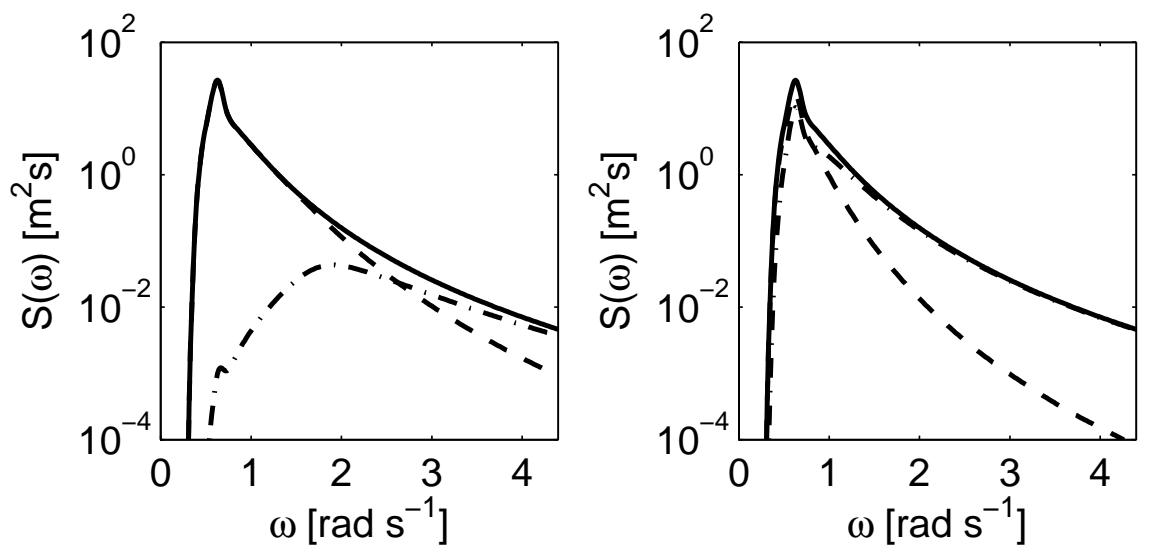

Figure 8: Example of input (solid curve), prediction (dashed curve) and prediction error (dash-dot curve) spectra in logarithmic scale. The distance to target location is set to $500 \mathrm{~m}$. The directional spread is set to $6^{\circ}$ for the plots on left and $30^{\circ}$ for the plots on the right side.

We have studied the convergence of prediction error variance at distance $T D / \lambda_{p} \approx 8$ by gradually increasing $I L$. A stopping criterion has been imposed in the algorithm when a given threshold is achieved by the absolute difference between successive prediction errors. The result is shown in Fig. 10 where the prediction error variances according to Eq. 7, are seen against the transverse distance denoted by $I L$ in Fig. 6. It indicates that the required size of $I L$ increases with increasing directional spread (See also table 2 for further detail). However, increasing the size of $I L$ further does not significantly improve the results. The above consideration is linear and purely numerical. We now turn to laboratory experiments and nonlinear wave theory. 
(a)

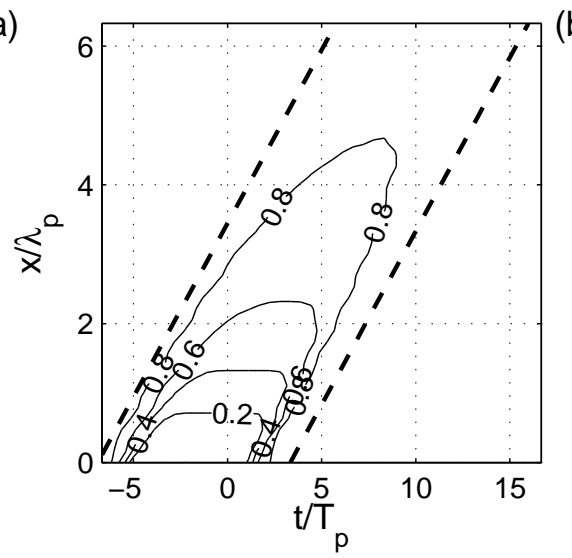

(b)

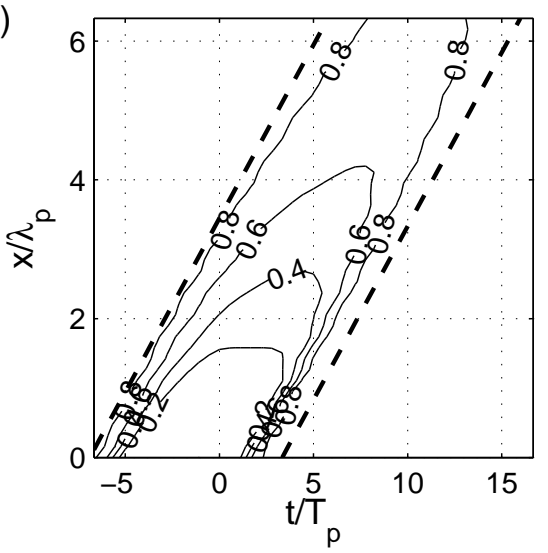

Figure 9: Prediction error variances from (a) simulation with single observation at $\mathbf{r}=(0,0)$ and (b) from simulation with three observations at $\mathbf{r} \in\{(0,-50 \mathrm{~m}),(0,0),(0,50 \mathrm{~m})\}$. The directional spread is set to $\sigma_{1}=30^{\circ}$ and the slope of the two slanted dashed lines corresponds to the magnitude of the LWT group velocity at the spectral peak.

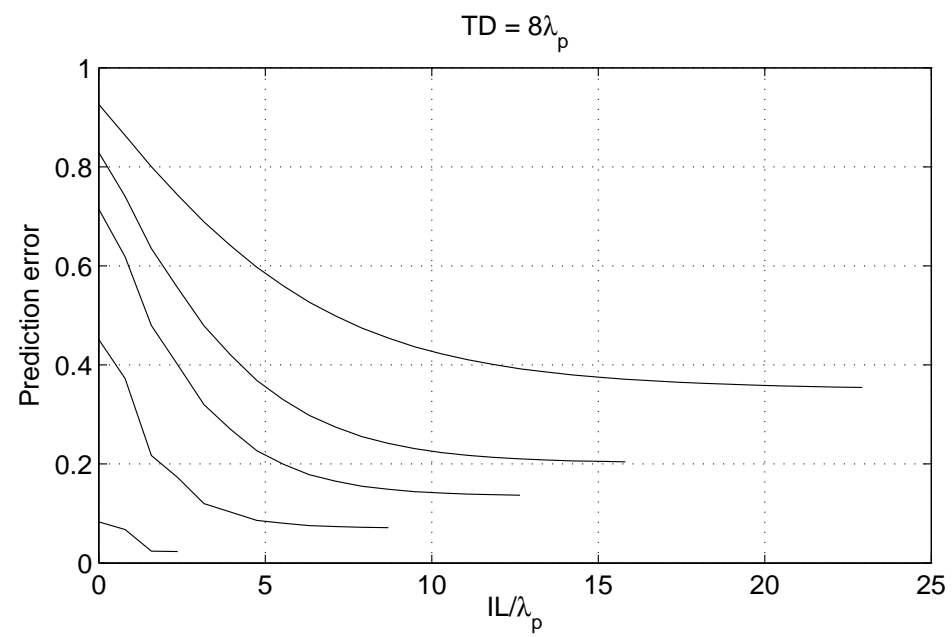

Figure 10: Required input length $I L$ for prediction at a target with distance $T D / \lambda_{p}=8$. Curves from bottom to top have $\sigma_{1}=6^{\circ}, 12.5^{\circ}, 19^{\circ}, 25^{\circ}, 37.5^{\circ}$. 
Table 2: Estimated ratio between $I L$ and $T D$ for optimal prediction in different sea states

\begin{tabular}{lccccc}
\hline$\sigma_{1}\left[^{\circ}\right]$ & 6 & 12.5 & 19 & 25 & 37 \\
\hline$I L / T D$ & 0.3 & 1.1 & 1.6 & 2 & 2.9 \\
$\theta_{p}\left[^{\circ}\right]$ & 17 & 58 & 77 & 90 & 111
\end{tabular}

\subsection{Laboratory experiments}

Sample comparisons between short-crested sea experiments in the MARIN basin and MNLS simulations are shown in Fig. 11, The comparisons are within a window moving with the magnitude of the LWT group velocity of the characteristic wave. The agreement between the experiments and simulations is quite good. The figure suggests much better comparison than the indicated error values would intuitively suggest. These error values are also dependent on the length of the sample time-trace we choose, and in most cases the normalized errors are greater than twice the corresponding mean absolute errors. As an example, from Fig. 11 the normalized prediction error at $x / \lambda_{c}=14.2$ is 0.41 with prediction time, $28 T_{c}$. The mean absolute error, in this case, is about 0.2. However, with shorter time-trace in the initial domain one may achieve a smaller normalized error without altering the physical comparison between prediction and the experiments.

Ensemble-averaged prediction errors from all experiments employing Eq. 21 are shown in Fig. 12 for all of the above prediction methods. The linear least squares model is fully empirical, it searches the required optimal time-shift between observation and prediction sites leading to a reasonable prediction of LWT group velocity. The agreement between prediction and experiment is perfect when the prediction site is quite close to the initial domain and becomes progressively worse when the prediction distance increases. The least squares prediction error is smaller than both linear theory and the NLS for bichromatic wave experiments, whilst in good agreement for the irregular wave experiments. For the short-crested sea experiments in the MARIN basin, the least squares 


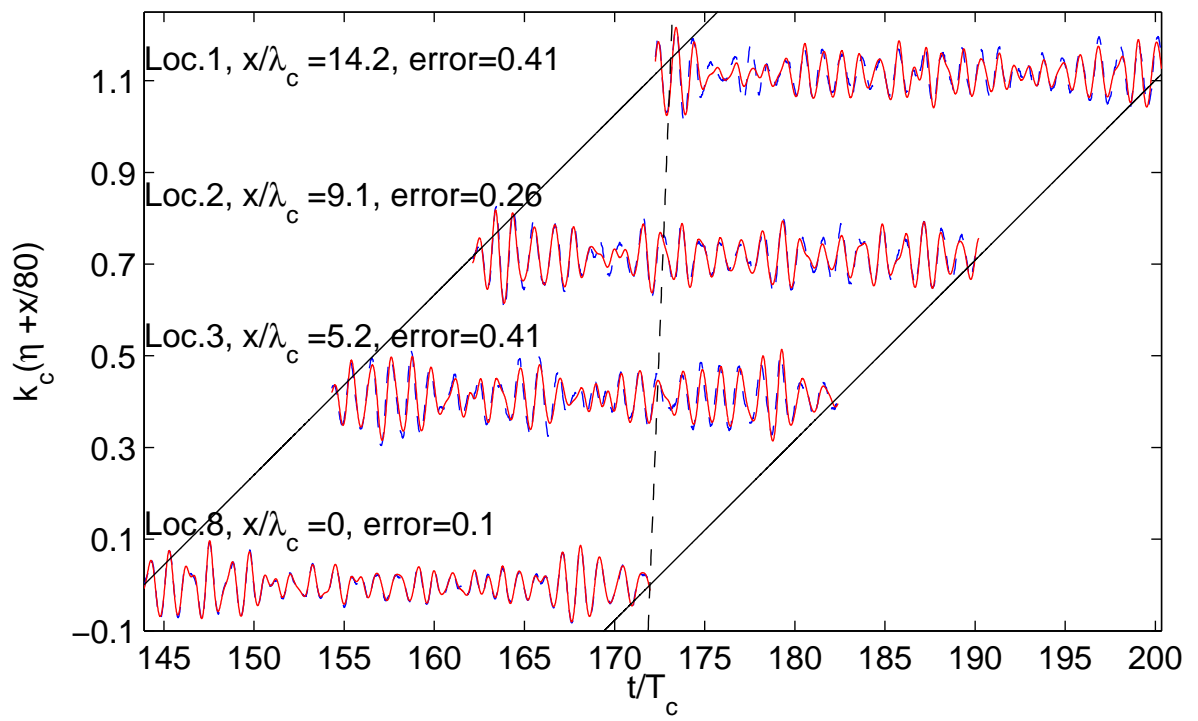

Figure 11: MARIN $\left(\sigma_{1}=12.5^{\circ}\right)$ : MNLS prediction with exact linear dispersion. Comparison between experiment (blue dashed curves) and prediction (red solid curves) at four locations. The two slanted lines have slope given by the magnitude of the LWT group velocity of the characteristic wave. The vertical dashed line separates the hind-cast and forecast. 
method is seen to provide the worst prediction. It is also found that the covariance based prediction does not improve the accuracy of predictions as expected. It gives exactly the same prediction as the LSQ. Due to truncation to narrow bandwidth, all Schrödinger equations do not in general reproduce the exact initial data, as a result one could expect a residual error at the initial condition. Except at the initial condition, both LDWP and LS produce virtually identical prediction. Although the NLS accounts for additional wave physics like nonlin375 ear increase in phase speed and modification of amplitudes within wave groups, it often over-predicts the maximum crest height observed in the experiments. This can lead to poor prediction, sometimes even worse than the linear wave theory as reported before. In most cases, the MNLS provides prediction with accuracy better than that obtainable both by NLS and linear wave prediction methods. Nevertheless, for the most short-crested waves with $\sigma_{1}=19^{\circ}$, it is hardly possible to identify difference between linear and nonlinear predictions, they all fail after a short propagation distances. This is mainly due to our lack of adequate input data in the transverse direction, and perhaps partly due to bandwidth limitation of the Schrödinger equations. However, we are not able to distinguish between the levels of influence from bandwidth limitation and input data inadequacy.

The MARIN experiments with $\sigma_{1}=12.5^{\circ}$ provide enough data to investigate the size of $I L$ relative to $T D$ required for an optimal prediction. The setting is similar to Fig. [6 with parameters and measurement locations specified in Fig. $4 \mathrm{~b}$, Four setups were investigated for accurate prediction of the downstream wave field. The normalized prediction errors between experiments and MNLS simulations are shown in Fig. 13 for the various cases. Obviously, measurements from location 8 alone are not enough to characterize the wave field. Measurements from locations 7-9 provide reliable prediction only up to about $6 \lambda_{c}$, whereas by incorporating measurements from locations 6 and 10, the prediction can be extended farther than $9 \lambda_{c}$ with good precision. Finally, measurements from all locations, i.e. 5-11, were found to furnish the amount of input data needed all the way out to $14 \lambda_{c}$. It may be noted that these val- 

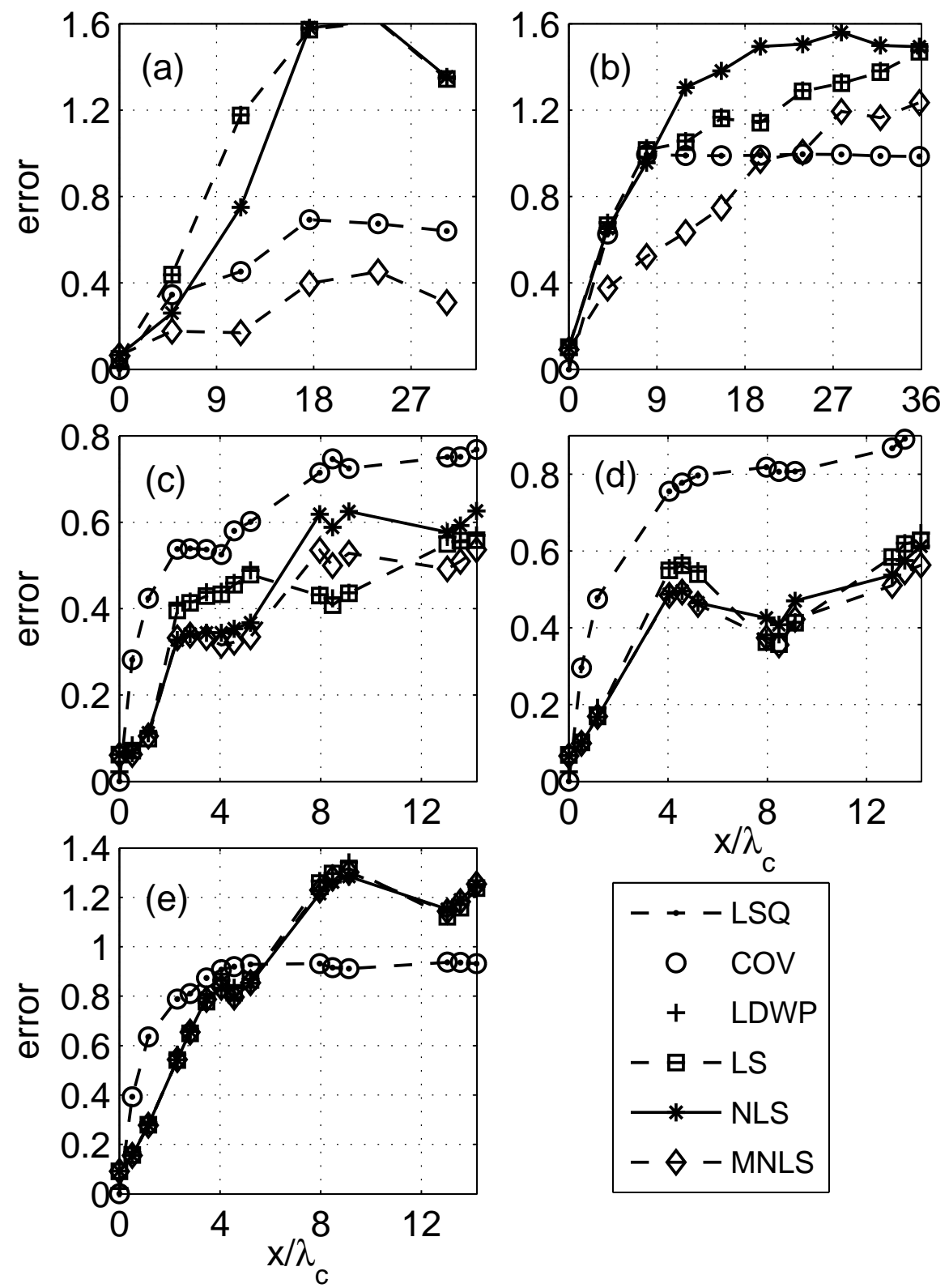

Figure 12: Marintek and MARIN wave data: Ensemble normalized prediction errors as a function of normalized propagation distance. (a) bichromatic long-crested waves, (b) irregular long-crested waves, (c) directional waves with $\sigma_{1}=6^{\circ}$, (d) directional waves with $\sigma_{1}=12.5^{\circ}$, (e) directional waves with $\sigma_{1}=19^{\circ}$. 


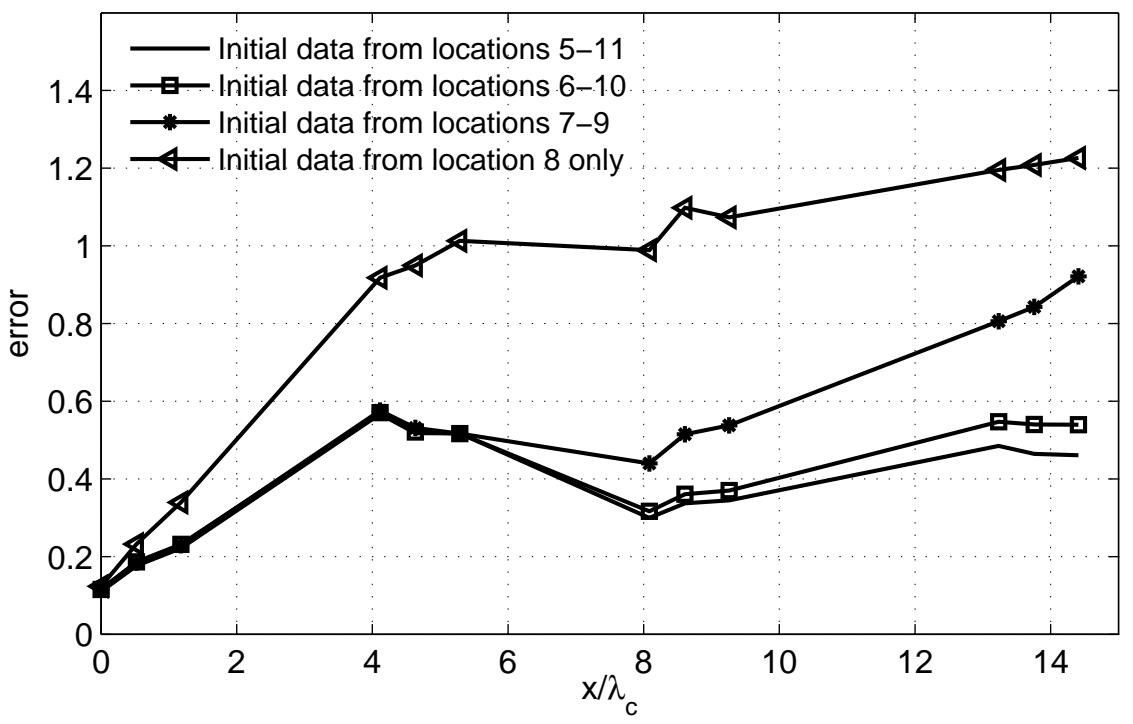

Figure 13: MARIN $\left(\sigma_{1}=12.5^{\circ}\right)$ : Normalized error of MNLS simulations as a function of normalized propagation distance at subsequent probes. The simulations are initialized by the leftmost probes of the locations indicated in the legend.

ues correspond approximately to the values obtained by the linear numerical experiments in Sec. 4.1. This means that, both in the numerical and in the laboratory experiments, the ratio between $I L$ and $T D$ is approximately equal to one for waves with $\sigma_{1}=12.5^{\circ}$.

Figures 14and 15 show comparisons of all experiments with LDWP and with MNLS simulations respectively. We compare ensemble-average prediction errors for bichromatic waves $\left(\sigma_{1}=0\right)$, irregular waves $\left(\sigma_{1}=0\right)$ and short-crested seas with directional spread $\sigma_{1}=6^{\circ}, 12.5^{\circ}$ and $19^{\circ}$. In order to ease the interpretation, in all short-crested sea simulations, except the one denoted by (II), we use initial data from locations 7-9. We observe large variations between these experiments, mainly attributed to differences in wave directionality. In both simulations, the prediction errors for the short-crested waves are situated above the prediction error for the irregular waves with the bichromatic waves below. The bichromatic experiments yield the smallest prediction error providing the 


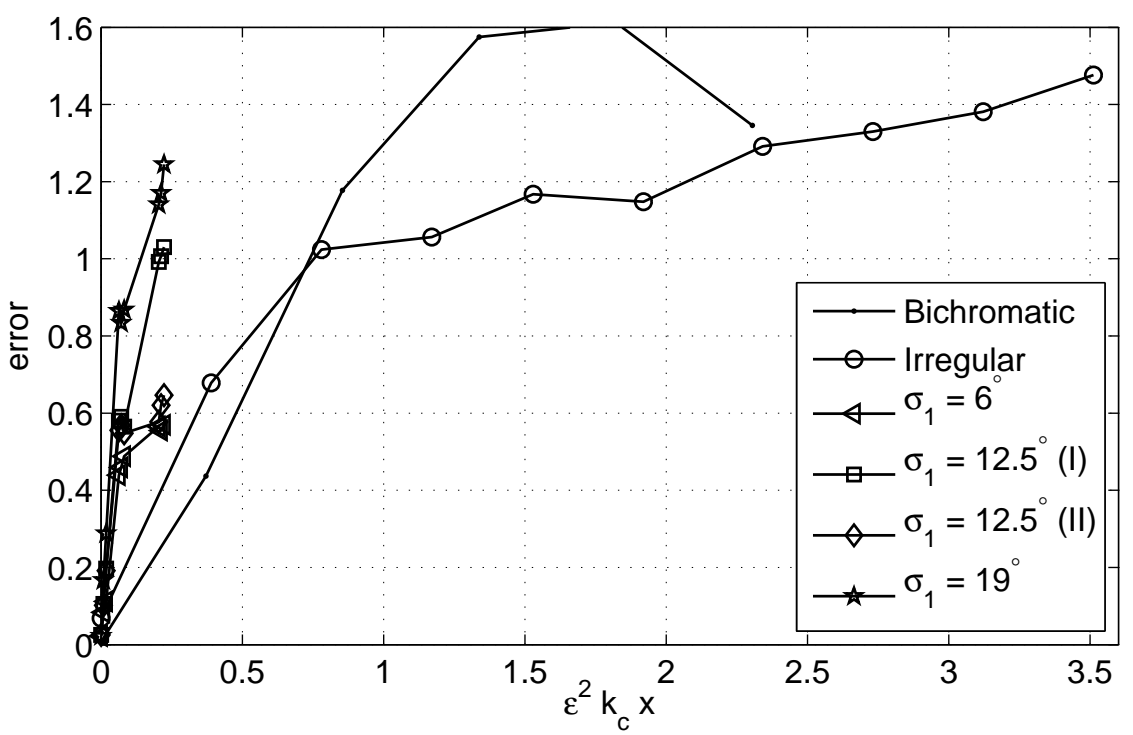

Figure 14: Marintek (lower two) and MARIN (upper four) experiments: LDWP ensembleaveraged prediction error as a function of normalized propagation distance. The prediction error is the lowest for the unidirectional bichromatic waves and the highest for the most shortcrested waves. Prediction errors are computed at probes A5, E5 and K5 of locations 8, 3 and 1. (I) and (II) denote inadequate and adequate input data respectively. 
longest prediction horizon. These waves are considerably more narrow-banded than the other, making them more suitable for MNLS-type simulations. For short-crested waves, the directional spread has a negative impact on the accuracy of prediction. We also recall that lack of sufficient input data for some of the above experiments lowers the accuracy (see also Fig. 16 for enhanced view of results from the short-crested sea experiments in the MARIN basin).

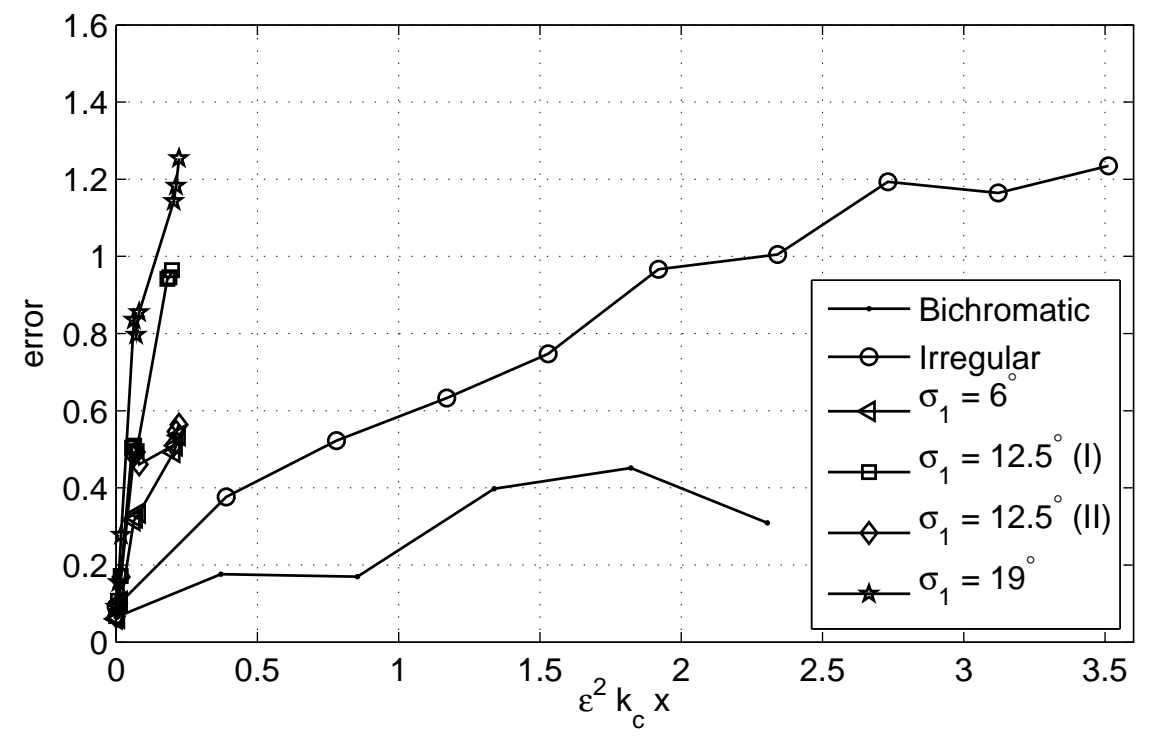

Figure 15: Marintek (lower two) and MARIN (upper four) experiments: MNLS ensembleaveraged prediction as a function of normalized propagation distance. The prediction error is the lowest for the unidirectional bichromatic waves and the highest for the most short-crested waves. (I): inadequate input data, (II): adequate input data.

Moreover, Fig. 15 reveals that the MNLS prediction range for long-crested irregular waves is more than twice as large as the LDWP range shown in Fig. 14, whereas for short crested seas no major improvement is observed. We believe that the combination of peak period and steepness in the MARIN experiments rendered the size of the basin too small to show a possible improved prediction horizon for the modified nonlinear Schrödinger equation in this case.

For the long-crested irregular wave experiments in the Marintek towing tank, 


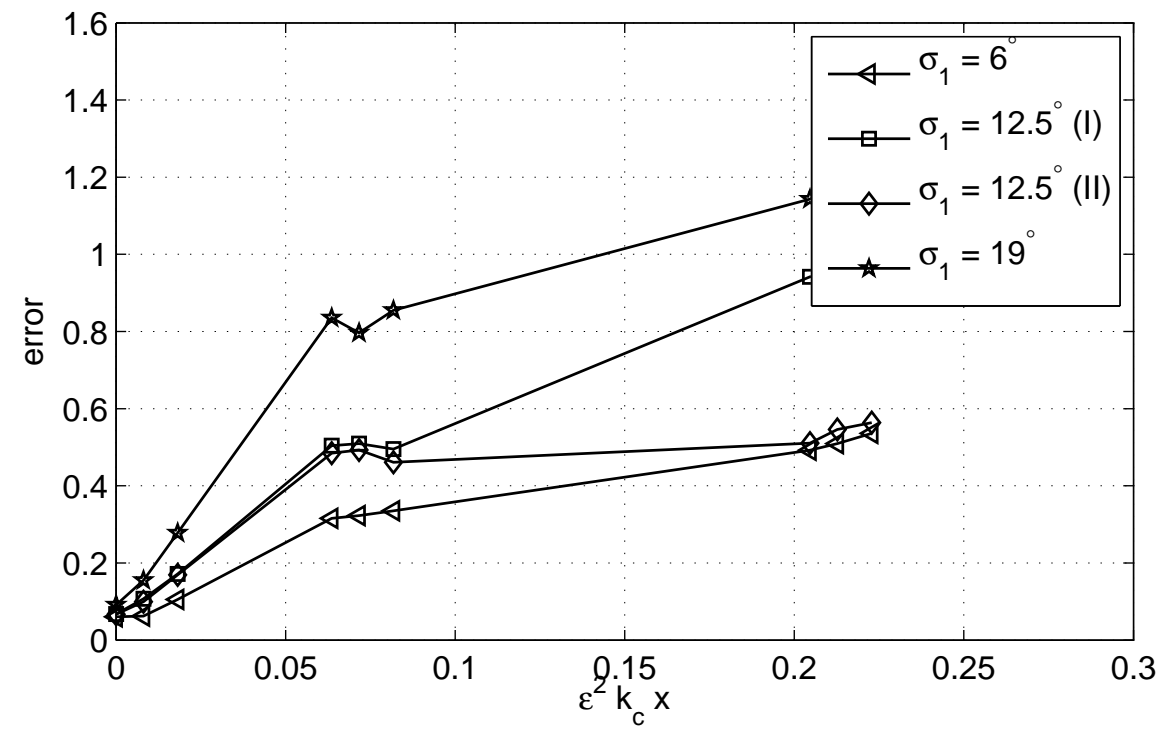

Figure 16: MARIN experiments: MNLS ensemble-averaged prediction error as a function of normalized propagation distance. Prediction errors are computed at probes A5, E5 and K5 of locations 8, 3 and 1. (I) and (II) are as described in Fig. 15

reliable prediction can be performed up to $\epsilon^{2} k_{c} x \approx 2.4$. For $\epsilon=0.1$, the MNLS prediction distance is about 40 characteristic wavelengths.

Based on short-crested wave experiments in the MARIN basin we anticipate a prediction range by $\epsilon^{2} k_{c} x=1$, which for $\epsilon=0.05$ would correspond to 65 characteristic wavelengths. However, in order to make definite conclusion about this prediction range, it is necessary to extend the validation effort at least up to $\epsilon^{2} k_{c} x=1$.

\section{Conclusions}

In this paper we have presented validation of various prediction models for long and short-crested waves in laboratory and numerical experiments. The modified nonlinear Schrödinger equation gives better prediction of the wave fields in the experiments for long fetch. Due to the structure of nonlinear interactions on deep water we anticipate the prediction range scales with $\epsilon^{2}$ where 
$\epsilon$ is the steepness of the wave field. The covariance based method is handy for numerical assessment when combined with linear wave theory.

The study indicates reliable nonlinear deterministic prediction can be performed up to $\epsilon^{2} k_{c} x=2.4$ for long-crested irregular waves. For short-crested waves we anticipate reliable nonlinear deterministic prediction up to $\epsilon^{2} k_{c} x=1$, provided there is sufficient input data to initialize the model. We have examined the amount of transversal input data necessary for the upstream wave fields. Both numerical and basin experiments suggest that required input data extension in the transverse direction increases with increasing directional spread. Acknowledgments. We would like to acknowledge the constructive comments by the referees. For the present study use has been made of the extensive 3D wave measurements conducted by Maritime Research Institute Netherlands (MARIN) in Wageningen as part of the On Board Wave and Motion Estimator (OWME) JIP. The authors would like to thank MARIN and the participants in the OWME JIP (Gusto, Kongsberg, SBM Seaflex, Sirehna, Statoil and Total) for making the data available for this study. Laboratory data from Marintek in Trondheim was kindly made available by Carl Trygve Stansberg. The Marintek towing tank experiment was funded by the Research Council of Norway. The Marintek ocean basin experiment was part of a joint project between Instituto Superior Tecnico and Marintek, partly supported by the European Union (ERBFMGECT980135). This research has been funded by the Research Coun460 cil of Norway, and the University of Oslo through projects RCN 225933 and RCN 214556.

\section{References}

Brillinger, D.R., 2001. Time Series: Data Analysis and Theory. Classics in Applied Mathematics, Society for Industrial and Applied Mathematics.

465 Brinch-Nielsen, U., Jonsson, I.G., 1986. Fourth order evolution equations and stability analysis for stokes waves on arbitrary water depth. Wave Motion 8, $455-472$. 
Burg, J.P., Luenberger, D.G., Wenger, D.L., 1982. Estimation of structured covariance matrices. Proceedings of the IEEE 70, 963-974.

Clauss, G.F., Kosleck, S., Testa, D., 2009. Critical situations of vessel operations in short-crested seas-forecast and decision support system, in: OMAE 2009-28th Intl Conference on Offshore Mechanics and Arctic Engineering, Honolulu, Hawaii, USA.

Clauss, G.F., Testa, D., Kolesck, S., Hessner, K., 2008. Forecast of critical situations in short-crested seas, in: OMAE 2008-27th Intl Conference on Offshore Mechanics and Arctic Engineering, Estoril, Portugal.

Clauss, G.F., Testa, D., Kosleck, S., Stück, R., 2007. Forecast of critical wave groups from surface elevation snapshots, in: OMAE 2007-26th Intl Conference on Offshore Mechanics and Arctic Engineering, San Diego, USA.

Ducrozet, G., Bonnefoy, F., Touz, D.L., Ferrant, P., 2012. A modified high-order spectral method for wavemaker modeling in a numerical wave tank. European Journal of Mechanics - B/Fluids 34, 19 - 34.

Dysthe, K.B., 1979. Note on a modification to the nonlinear schrödinger equation for application to deep water waves. Proceedings of the Royal Society of London. A. Mathematical and Physical Sciences 369, 105-114.

Dysthe, K.B., Trulsen, K., Krogstad, H.E., Socquet-Juglard, H., 2003. Evolution of a narrow-band spectrum of random surface gravity waves. Journal of Fluid Mechanics 478, 1-10.

Fusco, F., Ringwood, J.V., 2012. A study of the prediction requirements in realtime control of wave energy converters. IEEE Transactions on Sustainable Energy 3, 176-184.

Goda, Y., 2010. Random Seas and Design of Maritime Structures. Advanced series on ocean engineering, World Scientific. 
Golub, G.H., Van Loan, C.F., 2013. Matrix computations. Johns Hopkins Studies in the Mathematical Sciences, Johns Hopkins University Press.

Hasimoto, H., Ono, H., 1972. Nonlinear modulation of gravity waves. Journal of the Physical Society of Japan 33, 805-811.

Kahma, K., Hahuser, D., Krogstad, H.E., Lehner, S., Monbaliu, J.A.J., Wyatt, L.R., 2005. Measuring and analysing the directional spectra of ocean waves. volume EUR 21367, ISBN 92-898-0003-8.

Lo, E.Y., Mei, C.C., 1985. A numerical study of water-wave modulation based on a higher-order nonlinear Schrödinger equation. Journal of Fluid Mechanics $150,395-416$.

Lo, E.Y., Mei, C.C., 1987. Slow evolution of nonlinear deep water waves in two horizontal directions: A numerical study. Wave Motion 9, $245-259$.

Longuet-Higgins, M.S., Cartwright, D.E., Smith, N.D., 1963. Observations of the directional spectrum of sea waves using the motion of a floating buoy, in: Ocean Wave Spectra, National Academy of Sciences. Prentice-Hall. pp. $111-136$.

Mardia, K.V., 1972. Statistics of directional data. Probability and Mathematical Statistics a Series of Monographs and Textbooks, Academic Press.

Naaijen, P., Huijsmans, R.H.M., 2010. Real time estimation of ship motions in short crested seas, in: OMAE 2010-29th Intl. Conference on Offshore Mechanics and Arctic Engineering, Shanghai, China.

515 Naaijen, P., Trulsen, K., Blondel-Couprie, E., 2014. Limits to the extent of the spatio-temporal domain for deterministic wave prediction. International Shipbuilding Progress 61, 203-223.

Naaijen, P., Van Dijk, R.R.T., Huijsmans, R.H.M., El-Mouhndiz, A.A., 2009. Real time estimation of ship motions in short crested seas, in: OMAE 2009${ }_{520}$ 28th Intl. Conference on Offshore Mechanics and Arctic Engineering, Honolulu, Hawaii, USA. 
Nocedal, J., Wright, S.J., 2000. Numerical optimization. Springer New York.

Øksendal, B., 2003. Stochastic Differential Equations: An Introduction with Applications. Hochschultext/Universitext, Springer.

${ }_{525}$ Shemer, L., Dorfman, B., 2008. Experimental and numerical study of spatial and temporal evolution of nonlinear wave groups. Nonlinear Processes in Geophysics 15, 931-942.

Shemer, L., Goulitski, K., Kit, E., 2007. Evolution of wide-spectrum unidirectional wave groups in a tank: an experimental and numerical study. European Journal of Mechanics - B/Fluids 26, 193-219.

Shemer, L., Jiao, H., Kit, E., 1998. Nonlinear wave group evolution in deep and intermediate-depth water: Experiments and numerical simulations.

Shemer, L., Jiao, H., Kit, E., Agnon, Y., 2001. Evolution of a nonlinear wave field along a tank: experiments and numerical simulations based on the spatial Zakharov equation. J. Fluid Mechanics 427, 107-129.

Shemer, L., Kit, E., Jiao, H., 2002. An experimental and numerical study of the spatial evolution of unidirectional nonlinear water-wave groups. Physics of Fluids 14, 3380-3390.

Simanesew, A., Krogstad, H.E., Trulsen, K., Nieto Borge, J.C., 2016. Development of frequency-dependent ocean wave directional distributions. Applied Ocean Research 59, $304-312$.

Socquet-Juglard, H., Dysthe, K., Trulsen, K., Harald, E.K., Liu, J., 2005. Probability distributions of surface gravity waves during spectral changes. Journal of Fluid Mechanics 542, 195-216.

545 Stansberg, C.T., 1993. Propagation-dependent spatial variations observed in wavetrains generated in a long wave tank. Technical Report MT49 A93-0176. Marintek. 
Stansberg, C.T., 1995. Spatially developing instabilities observed in experimental bichromatic wave trains, in: A. J. Grass, (ed.), 26th IAHR Congress (HYDRA 2000), pp. 180-185.

Stansberg, C.T., 1998. On the nonlinear behaviour of ocean wave groups, in: Proceedings of the 3rd Intl. Symposium on Ocean Wave Measurement and Analysis-WAVES'97 (ASCE), pp. 1227-1241.

Toffoli, A., Gramstad, O., Trulsen, K., Monbaliu, J., Bitner-Gregersen, E., Onorato, M., 2010. Evolution of weakly nonlinear random directional waves: laboratory experiments and numerical simulations. Journal of Fluid Mechanics $664,313-336$.

Trulsen, K., 1998. Crest pairing predicted by modulation theory. Journal of Geophysical Research: Oceans 103, 3143-3147.

Trulsen, K., 2003. Real time deterministic ocean wave forecasting using weakly nonlinear wave evolution theory, in: MekIT'03, Second National Conference on Computational Mechanics, Trondheim. Tapir Akademisk Forlag., pp. 371390 .

Trulsen, K., 2005. Spatial evolution of water surface waves, in: Proceedings of the Fifth Intl. Symposium on Ocean Wave Measurement and AnalysisWAVES 2005, Madrid, Spain.

Trulsen, K., Dysthe, K.B., 1997. Frequency downshift in three dimensional wave trains in a deep basin. Journal of Fluid Mechanics 352, 359-373.

Trulsen, K., Kliakhandler, I., Dysthe, K.B., Velarde, M.G., 2000. On weakly nonlinear modulation of waves on deep water. Physics of Fluids 12, 24322437.

Trulsen, K., Stansberg, C.T., 2001. Spatial evolution of water surface waves: Numerical simulation and experiment of bichromatic waves, in: Proceedings of the Eleventh International Offshore and Polar Engineering Conference, pp. 575 71-77. 
Wu, G., 2004. Direct simulation and deterministic prediction of large-scale nonlinear ocean wave-field. Ph.D. thesis. Massachusetts Institute of Technology.

Wu, G., Liu, Y., Yue, D.K.P., 2007. Ocean wave prediction using large-scale phase-resolved computations, in: DoD High Performance Computing Modernization Program Users Group Conference, 2007, pp. 449-454.

Xiao, W., Liu, Y., Yue, D.K.P., 2009. Ocean wave prediction using largescale phase-resolved computations, in: DoD High Performance Computing Modernization Program Users Group Conference (HPCMP-UGC), 2009, pp. $278-284$.

Zakharov, V.E., 1968. Stability of periodic waves of finite amplitude on the surface of a deep fluid. Journal of Applied Mechanics and Technical Physics 9, 190-194. 